\title{
Le « contact virtuel » entre un esprit et un corps et l'action à distance
}

Une notion apparaît dans divers textes scolastiques de théologie ou de philosophie, le contactus virtualis, à propos de deux questions : l'action de l'esprit sur un corps et l'action à distance. Ce double contexte invite à s'interroger sur les manières dont l'action d'une substance spirituelle sur un corps pouvait être pensée dans le cadre d'une philosophie péripatéticienne imposant un contact entre l'agent et le patient.

\section{De l'esprit au corps : le contact virtuel selon Thomas d'Aquin}

Thomas d'Aquin à plusieurs reprises se réfère à la notion de contact virtuel pour expliquer la modalité propre à l'action d'une substance spirituelle (démon, ange, Dieu ou âme) ${ }^{1}$.

\section{Le démon et le corps}

Dans les tardives Quaestiones disputatae de malo (datées de 1269-1270), au dixième article de la seizième question, Thomas d'Aquin demande « si les démons peuvent mouvoir localement les corps ». Parmi ses objections, deux sont significatives pour le présent propos. La première affirme qu'une substance spirituelle ne peut mouvoir un corps que si elle la vivifie (comme le fait l'âme). La seconde consiste à soutenir que le démon, substance spirituelle, n’a rien de commun avec un corps et qu'il ne peut donc y avoir aucune action. Sur le rôle éventuel des démons dans le mouvement local, la position de Thomas est bien connue. Le mouvement local, explique-t-il, est à la fois le premier de tous les mouvements et celui qui provoque la plus faible modification dans le sujet. Les substances spirituelles étant par nature plus élevées que les corps, elles peuvent donc produire le

1 Requêtes dans l'Index Thomisticus : www.corpusthomisticum.org.

Nicolas Weill-Parot, École Pratique des Hautes Études, EPHE PSL 
premier des mouvements, le mouvement local : les anges sur les corps célestes ; les démons sur les corps inférieurs. Comme le mouvement local est celui qui est du plus faible effet sur le sujet, il peut être produit de manière immédiate. L'Aquinate en déduit donc que les démons produisent immédiatement un mouvement local dans un corps inférieur.

Il répond à la première objection mentionnée ici en expliquant que si l'âme ne peut mouvoir localement que par la vivification du corps, c'est parce qu'elle « occupe le degré le plus bas dans l'ordre des substances spirituelles »². Et sa réponse à l'autre objection est ainsi formulée :

Entre les démons et les corps il n'y a pas de contact corporel, mais virtuel, qui requiert néanmoins une convenance de proportion entre le moteur et le mobile ${ }^{3}$.

Il n'en dit pas plus ici sur la nature de ce contact virtuel qui rend compte de la capacité des démons à mouvoir localement les corps (seul mouvement que les démons peuvent produire).

Ce qu'il faut retenir pour le moment, c'est la motivation première de Thomas d'Aquin : respecter l'exigence de contact entre l'agent et le patient imposée par la philosophie naturelle aristotélicienne apparemment pour tout agent et tout patient quelle que soit sa nature, corporelle ou spirituelle. Dans l'objection auquel cet argument répond, Thomas s'était référé expressément au De genera-

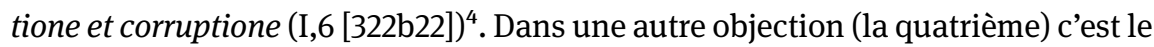
passage clef du livre VII de la Physique d'Aristote où est énoncé l'axiome « contiguïste » selon lequel « le moteur et le mû sont ensemble » qui est invoqué5.

Aux yeux de Thomas postuler un « contact virtuel » entre le démon et le corps c'est surmonter l'obstacle d'une différence de nature entre une substance

2 Thomas de Aquino, Quaestiones disputatae de malo, éd. Commissio Leonina, Rome-Paris 1982, 327b : " Ad secundum dicendum quod anima humana infimum gradum tenet in ordine substantiarum spiritualium, unde non habet uirtutem mouendi corpus, etiam localiter, nisi proportionatum sibi per hoc quod est uiuificatum ab ea».

3 Ibid. : «Ad tertium dicendum quod demonis ad corpus non est contactus corporalis set uirtualis; qui quidem requirit conuenientiam proportionis mouentis ad mobile ».

4 Ibid., 326b : « [3.] Preterea. Omnis actio est per contactum, ut dicitur in I De generatione. Non uidetur autem quod possit esse aliquis contactus demonis ad corpora : quia nichil habet cum eis commune. Cum ergo mouere localiter sit quoddam agere, uidetur quod demones non possint corpora localiter mouere $»$.

5 Ibid., 326b : « [4]. Preterea. Si demones possent aliqua corpora mouere localiter, maxime possent mouere localiter corpora celestia, que propinquiora sunt eis in ordine nature. Set corpora celestia mouere non possunt ; quia cum movens et motum sint simul, ut dicitur in VII Phisicorum, sequeretur quod demones sint in celo ; quod neque secundum nos est uerum, neque secundum Platonicos. Ergo multo minus possunt mouere alia corpora ». Cf. Aristote, Physique, VII.2, 243a32-35. 
spirituelle et un substance corporelle, en réintégrant le cadre contiguïste aristotélicien. Cette modalité, qui apparemment respecte cette injonction contiguïste aux yeux de Thomas, semble donc le propre du contact externe entre une substance spirituelle et un corps.

Pour autant qu'on puisse en juger d'après les différentes recherches réalisées dans l'Index thomisticus, cette notion (tactus virtualis, contactus virtualis ou contactus secundum virtutem) apparaît chez Thomas d'Aquin essentiellement (voire uniquement) lorsqu'il est question de l'action d'une substance spirituelle sur un corps.

\section{Les anges et le lieu}

Thomas évoque le contact virtuel à propos des anges lorsqu'il aborde la question de leur localisation. Il s'agit, en somme, de comprendre la présence d'un ange en un lieu alors que, en tant que substance incorporelle, il ne peut être circonscrit en un lieu comme un localisé par un corps enveloppant ${ }^{6}$. Dans le onzième Quodlibet (qui appartient au groupe de questions quodlibétiques datées du séjour parisien de 1256 à 1259), quand il s'agit de savoir « si le mouvement des anges est instantané » ${ }^{7}$, il affirme que le mouvement de l'ange est accompli « selon divers contacts virtuels entre l'ange et les divers lieux $\|^{8}$. Dans le très tardif De substantiis separatis, daté de 1272-1273, Thomas expose que les anges ne sont pas mus corporellement en un lieu, mais « suivant une succession de contacts virtuels » avec les différents lieux 9 .

\footnotetext{
6 Voir notamment : T. Suarez-Nani, Les Anges et la Philosophie : subjectivité et fonction cosmologique des substances séparées à la fin du XIIIe siècle, Paris 2002.

7 Thomas de Aquino, Quaestiones de quolibet, éd. R.-A. Gauthier, éd. Commissio Leonina, RomeParis 1996, Quodl. XI, q. 4, art. unicus, 156a : «Circa angelos quesitum fuit de motu angeli, utrum scilicet motus Angeli sit in instanti? ».

8 Ibid., 157a : co. : "Sic igitur due sunt dispositiones mutationum corporalium; sed neutrum horum potest esse in motu angeli. Cum enim motus angeli attendatur secundum diuersos contactus uirtuales angeli ad diuersa loca, qui quidem contactus non sunt continui, sequitur quod motus angeli non sit continuus, et ita tempus quod proprie mensurat motum angeli, non est continuum, cum continuitas temporis sit ex continuitate motus, ut dicitur in IV Physicorum [...] ». 9 Thomas de Aquino, De substantiis separatis, cura et studio Fratrum praedicatorum, Rome 1969, cap. 19, 76a : «Ex quibus omnibus datur intelligi quod angeli non sunt in loco corporali modo, sed quodam modo spirituali. Et quia eodem modo competit alicui moveri in loco et esse in loco, per consequens neque corporali modo angeli moventur in loco ; sed motus eorum qui exprimitur in Scripturis, si referatur ad locum corporalem, est accipiendus secundum successionem virtualis contactus ad loca diversa ; vel est accipiendus secundum mysticam intelligentiam, sicut Dionysius, IV cap. de divinis nominibus dicit, quod "moveri dicuntur divinae mentes circulariter quidem unitae illuminationibus pulchri et boni ; in directum autem quando
} 


\section{Dieu et les corps}

La notion de contact virtuel apparaît aussi pour la question du contact entre Dieu et les créatures terrestres. Dans la prima pars de la Somme théologique, Thomas explique :

Comme [Dieu] est incorporel, il ne touche ni n'est touché. Mais selon un contact virtuel, il touche néanmoins les créatures en les mouvant, mais il n'est pas touché [par elles], parce que la vertu naturelle d'aucune créature ne peut l'atteindre ${ }^{10}$.

Ce contact virtuel est aussi convoqué dans la tertia pars de la Somme théologique [III, q. 56, art. 1], lorsque Thomas explique que la résurrection du Christ est cause efficiente de notre résurrection par la vertu divine qui « atteint par sa présence tous les lieux et tous les temps »; « un tel contact virtuel », précise-t-il, " suffit à la raison de cette efficience ». Comme l'écrit Martín Echavarría, le contact virtuel fonde ici chez Thomas une sorte d'ubi virtuel qui permet de dire que la « créature spirituelle » est « en ce sur quoi elle agit, mais d'une autre manière ${ }^{11}$.

\section{L’âme et le corps ?}

Dans la Quaestio disputata de spiritualibus creaturis, datée de 1267-1268, dans sa réponse à la question « la substance spirituelle qu'est l'âme humaine est-elle unie au corps par un intermédiaire ? », Thomas se réfère à l'hypothèse de certains (hypothèse qu'il rejette) selon laquelle la présence d'une pluralité d'intermédiaires serait justifiée par le fait que « l'âme rationnelle » serait « unie seulement par un contact virtuel, comme moteur $»^{12}$. Pour Thomas, il n'en est rien : elle est unie au corps comme une forme.

procedunt ad subiectorum providentiam, oblique autem quando providentes minus habentibus inegressibiliter manent" circa Deum. Ex his igitur manifestum est quid circa conditionem spiritualium substantiarum, id est angelorum, sacri doctores tradiderint, asserentes eos incorporeos et immateriales esse ".

10 « [...] Secundum igitur primum contactum, Deus, cum sit incorporeus, nec tangit nec tangitur. Secundum autem virtualem contactum, tangit quidem movendo creaturas, sed non tangitur, quia nullius creaturae virtus naturalis potest ad ipsum pertingere » (Thomas de Aquino, Summa theologiae, Milan 1962, rééd. 1988, Ia, q. 105, art. 2, ad 1, 490).

11 M. Echavarría, «La cantitad virtual (quantitas virtualis) según Tomás de Aquino », Logos. Anales del Seminario de Metafísica 46 (2013), 235-259 (ici : 247-248).

12 Thomas d'Aquin, Les créatures spirituelles, texte latin de l'édition Léonine, trad. et notes J.-B. Brenet, Paris 2010, 279, note 71 (évocation du contactus virtutis). «Si enim anima rationalis unitur corpori solum per contactum uirtualem, ut motor, ut aliqui posuerunt, nichil prohibebat 
Dans le reste du corpus thomiste, on trouve plusieurs références à ce type de contact. Il intervient toujours pour caractériser le contact entre une substance incorporelle et un corps. Le Lexicon of St Thomas Aquinas de Deferrari considère contactus ou tactus virtualis ou virtutis (« contact de pouvoir ou d'opération ») comme un synonyme de contactus spiritualis. Ce type de contact s'oppose au contactus/tactus quantitatis seu molis ${ }^{13}$. En revanche, la question de la distance n'est pas souvent mise en exergue dans ce cadre. Elle est au contraire première chez plusieurs auteurs du XIII ${ }^{\mathrm{e}}$ siècle, qui envisagent cette fois l'action dans le sens inverse : celle d'un corps sur une « substance spirituelle », plus précisément sur une faculté de l'âme.

\title{
2 Du corps vers l'esprit
}

\section{Un commentaire anglais de la Physique}

Un commentaire anglais anonyme à la Physique d'Aristote, daté par Silvia Donati d'entre les années 1250 et le début des années 1270, aujourd'hui conservé à Sienne $^{14}$, aborde au livre VII la question des phénomènes apparemment à distance mais en définitive réintégrables au cadre contiguïste aristotélicien. Le contact entre le corps et l'âme est traité en ces termes :

\begin{abstract}
Des choses peuvent être ensemble doublement, soit par contact soit par absence d'intermédiaire (privatio medii), comme cela est apparu dans le cinquième livre [de la Physique] et c'est pourquoi il n'est pas nécessaire qu'ils soient ensemble par contact, ils sont cependant ensemble parce qu'il y a une absence d'intermédiaire ; et ainsi, cela est manifeste pour ce cas, puisque l'âme ne touche pas le corps et qu'entre le corps et l'âme ne s'interpose aucun intermédiaire. Par cela il est manifeste, en réponse à l'autre argument, que ce qui est désirable envoie une species vers ce qui désire soit vers l'âme désirante, et ainsi, il informe l'âme, dans cette information de l'âme ils se touchent virtuellement, et sont ainsi virtuellement ensemble ${ }^{15}$.
\end{abstract}

dicere quod sunt multa media inter animam et corpus [...] » (Thomas de Aquino, Quaestio disputata de spiritualibus creaturis, éd. J. Cos, Commissio Leonis XIII, Rome-Paris 2000).

13 R. J. Deferrari, Sister M. I. Barry, collab. I. McGuiness, A Lexicon of St. Thomas Aquinas Based on the Summa Theologica and Selected Passages of His Other Works, Baltimore Md. 1948, 226.

14 Sur la datation de ce commentaire : S. Donati, « Per lo studio dei commenti alla Fisica del XIII secolo. Commenti di probabile origine inglese degli anni 1250-1270 ca. Parte I », Documenti e studi sulla tradizione filosofica medievale 2/2 (1991), 361-441 (ici : 396-409).

15 « [...] aliqua possunt esse simul dupliciter, scilicet per contactum aut per privationem medii sicut patuit in quinto / et (?) ideo non oportet quod sint simul per contactum, sunt tamen simul per hoc quod est privacio medii et sic patet ad hoc cum anima non tangat corpus sed inter cor- 
L'auteur renvoie manifestement à Physique V,3 [226b19-227b4] et se réfère à la doctrine de la perspectiva des species, ces simulacres considérés en eux-mêmes généralement comme immatériels, mais traversant l'intermédiaire en empruntant à ce dernier sa corporéité ${ }^{16}$.

\section{Pierre Auriol}

Dans une tout autre direction de recherche, Jean-Baptiste Brenet a relevé plusieurs utilisations de la notion de « contact virtuel » pour penser ce qu'il appelle l'« unité noético-somatique » chez des auteurs comme Jean de Jandun, Godrefoid de Fontaines ou le franciscain Pierre Auriol (v. 1280-1322). Il suppose même que la suggestion pourrait se retrouver dans le Grand Commentaire d'Averroès au De anima, où ce dernier montre que " l'âme et le corps forment un être un dans la mesure où ce ne sont pas deux êtres préalablement en acte », ce qui aurait pu donner l'idée de poser « une sorte de contact » entre « l'intellect séparé et le corps animé ». Pierre Auriol fait dériver « le principe individuel des pensées et des passions sensibles, qui est un et le même » précisément du « contact virtuel » qu'il y a entre «l'intellect » et le « corps ${ }^{17}$.

La lecture du passage pertinent dans le commentaire du deuxième livre des Sentences de Pierre Lombard par Pierre Auriol révèle cet intéressant (et amusant) passage :

Je pose que le vin de l'outre me touche de la manière que nous disons que la chose triste touche mon cœur, je demande : Qu'est-ce qui me touche le plus, l'outre de vin ou l'objet triste? Et il est certain que c'est le vin, parce que celui-ci le fait réellement, alors que l'objet

\footnotetext{
pus et animam non cadit medium. Per hoc patet ad aliud quod appetibile inmittit speciem ad appetens sive ad animam appetentem et sic informat animam, animam informando virtualiter tangunt se et sic virtualiter simul sunt » (ms. Sienne, bibl. comm. degli Intronati, L III 21, f. 79rb79va) (lu sur un microfilm).

16 Sur la doctrine de la multiplication des species et ses diverses implications, voir notamment : K.Tachau, Vision and Certitude in the Age of Ockham. Optics, Epistemology and the Foundation of Semantics 1250-1345, Leyde 1988 ; L. Spruit, "Species intelligibilis" from Perception to Knowledge. Vol. I : Classical Roots and Medieval Discussions, Leyde 1994.

17 J.-B. Brenet, « Moi qui pense, moi qui souffre. Le problème de l'identité du composé humain dans la riposte anti-averroïste de Pierre d'Auriole et Grégoire de Rimini », dans O. Boulnois (éd.), Généalogies du sujet de saint Anselme à Malebranche, Paris 2007, 151-169 (ici : 165-166 et note 3), cf. Averrois Cordubensis Commentarium magnum in Aristotelis De anima libros, éd. F. S. Crawford, Cambridge Mass. 1953, II,7, 139.
} 
triste le fait virtuellement : le vin, en effet, parvient à une plus grande union (unitatem), lui qui me touche, que l'objet triste, parce que l'objet triste qui me touche peut se trouver à Rome ${ }^{18}$.

Mais la notion de contact virtuel ne se limite pas au postulat d'une contiguïté spirituelle entre un esprit et un corps, on la trouve aussi utilisée dans les relations entre les corps, ce qui permettra en retour d'en éclairer les présupposés pour le contact entre esprit et corps. En premier lieu, on invoque ce contact entre les corps célestes et les corps terrestres qui leur sont distants.

\section{L'application de la notion de contact virtuel au contact entre corps céleste et corps terrestre}

\section{Richard de Clive}

Richard de Clive, un maître anglais, a rédigé son commentaire à la Physique vers 1270-1272. Lorsqu'il aborde la question « utrum movens et motum sint simul », il est confronté au contrexemple apparent constitué par les corps célestes qui semblent mouvoir à distance les corps inférieurs ${ }^{19}$. "Quelqu'un dira peut-être », écrit-il, « que le moteur et le mû sont ensemble virtuellement même si ce n'est pas corporellement $»^{20}$. Il retrouve cette même conjonction virtuelle dans le mouvement forcé de l'expulsion : «c'est une expulsion quand le moteur et le mû ne sont pas ensemble corporellement mais virtuellement, et ainsi la proposition est vraie pour tout mouvement ${ }^{21}$. Le cas du projectile est effectivement traité dans les commentaires de la Physique selon des modalités explicatives assez voisines de celles employées pour résoudre une action apparemment à distance comme l'attraction magnétique ou l'influence céleste.

18 (Petrus Aureolus, Commentariorum in secundum librum Sententiarum tomus secundus Rome 1605, II, dist. 17, 239a). « Pono quod vinum utri tangat me eo modo quo dicimus quod triste tangit cor. Quaero : Quid tangit me magis, utrum vini vel triste ? Et certum est quod vinum, quia illud realiter, triste virtualiter : ad maiorem enim unitatem venit vinum quod tangit me quam triste, quia triste quod tangit me potest esse Romae ».

19 Ricardus de Clive, Quaestiones super Physicam, VII: «Quero [...] utrum movens et motum sint simul ; et quod non videtur : corpus celeste movet hec inferiora, tamen non est simul cum hiis » (ms. Worcester, Cathedral Library, ms. Q. 13, f. 109ra) (lu sur un microfilm).

20 Ibid. : « Dicet aliquis forte quod movens et motum sunt simul virtualiter etsi non corporaliter".

21 Ibid., f. 109rb : « Expulsio est quando movens et motum non sunt simul corporaliter sed virtualiter et sic in omni motu vera est propositio ». 
Demeure un problème : comment peut-on affirmer que « le ciel et ces inférieurs sont ensemble virtuellement ", puisque, comme certains l'objectent, " une vertu ne peut exister sans substance » ? Richard de Clive répond que, bien évidemment, " une vertu ne peut exister sans support », mais qu'elle peut « exister sans la substance qui en est la cause ». "Une vertu propre » écrit-il, " ne peut exister sans son sujet propre comme la matière, elle peut cependant exister sans le sujet propre qui en est la cause, et c'est pourquoi la vertu du feu peut exister de cette manière ${ }^{22}$. L'action virtuelle permet de rendre compte de l'action du corps céleste sur le corps terrestre. Il s'agit bien d'un contact puisque entre la vertu céleste et le corps terrestre il y a une absence d'intermédiaire (medium). La vertu ne peut certes exister sans son substrat propre, elle peut en revanche exister séparée de la cause qui l'a produite.

\section{Jérôme Torrella}

Un autre exemple, pris dans la scolastique tardive, d'une mention d'un contact virtuel entre corps céleste et corps terrestre, et même entre deux corps terrestres, est offert dans l'Opus praeclarum de imaginibus astrologicis, achevé en 1496 par le médecin valencien Jérôme Torrella. Ce dernier s'interroge sur les raisons pour lesquelles des fils ayant servi à étrangler une vipère sont efficaces contre l'angine, comme le disent Galien et Avicenne. Il compare ce procédé à l'« image astrologique ", ce sceau talismanique fabriqué sous une constellation donnée et censée s'imprégner de l'influence naturelle des astres ${ }^{23}$.

Peut-être quelqu'un dira-t-il qu'intervient pour la guérison de l'angine un contact naturel et physique [entre le fil et le cou], nous devons dire que cela est vrai. Mais le contact du ciel avec l'image est virtuel, et il suffit à conserver la santé et à préserver de la maladie et à guérir la mauvaise santé du corps humain, puisque la qualité occulte est productrice de tels effets, tout comme les docteurs affirment que la pierre d'aigle portée par une

22 Ibid., f. 109rb. : « Ad primum argumentum dicendum quod celum est simul cum hiis inferioribus virtualiter. Et si diceret aliquis quod virtus non est sine substantia, dicendum quod virtus non potest esse sine deferente, tamen potest esse sine substantia causante. Sed tunc queritur, cum virtus ignis non potest esse sine igne, quia propria virtus non est sine subiecto proprio, et tunc virtus, ut videtur, non potest esse huiusmodi sine igne. Dicendum quod ista propositio dupliciter potest intelligi, hoc est, virtus ignis aut quia est in igne ut in subiecto, aut quia causatur ab igne ; propria virtus non potest esse sine proprio subiecto ut materia, tamen potest esse sine subiecto proprio causante; et ideo virtus ignis potest esse huiusmodi ».

23 N. Weill-Parot, Les " images astrologiques " au Moyen Âge et à la Renaissance. Spéculations intellectuelles et pratiques magiques $\left(X I I^{e}-X V^{e}\right.$ siècle), Paris 2002. 
femme enceinte empêche la fausse-couche à travers un contact virtuel avec le ciel et avec la matrice et les cotylédons ${ }^{24}$.

Le concept de « contact virtuel » permet donc de replacer dans la contiguïté des procédés thérapeutiques magiques ou bien merveilleux qui paraissent fonctionner suivant une action à distance. Alors que le fil de la vipère est en contact physique avec le cou de la vipère, en revanche l'« image astrologique » n'est avec sa source astrale qu'en contact virtuel ; la pierre d'aigle, quant à elle, est dans un double contact virtuel : avec la source de son efficience (les cieux, les astres) et avec la cible de son efficacité (la matrice et les cotylédons).

$\mathrm{Ni}$ Richard de Clive ni Jérôme Torrella ne remettent en cause le cadre contiguïste aristotélicien. Au contraire, sous leur plume, la notion de « contact virtuel » est là pour rétablir une contiguïté entre le moteur et le mobile. En revanche, l'interprétation d'un tel contact est différente chez plusieurs auteurs du tournant du $\mathrm{XIII}^{\mathrm{e}}$ et du XIV ${ }^{\mathrm{e}}$ siècle : chez eux, il tend vers une action à distance.

\section{Le contact virtuel entre les corps, une action à distance?}

\section{Duns Scot}

La question de l'action à distance chez Duns Scot est un sujet vaste et complexe que des spécialistes comme Francis J. Kovach ont analysée en détail. Duns Scot mentionne des exemples, déjà attestés ailleurs, comme des cas d'attraction apparemment à distance : ainsi, la manière dont le soleil engendre sous la terre des plantes et des animaux ou l'engourdissement de la main du pêcheur quand il prend un poisson torpille dans son filet sans que le filet en soit affecté.

Les agents naturels agissent en général par un double contact " par la substance » et « par le pouvoir» (ou la vertu). Pourtant certains ont la capacité d'agir uniquement par la vitrtus et non par la substance, en somme ils agissent

24 Hieronymus Torrella, Opus praeclarum de imaginibus astrologicis, éd. N. Weill-Parot, Florence 2008, IV, 205 : « Dicet fortasse aliquis interuenire ad curationem squinantie contactum naturalem et phisicum, dicamus hoc verum esse. Caeli autem ad imaginem contactus est virtualis, qui sufficit ad sanitatem conseruandam et ab egritudine praeseruandum atque curandum aduersam corporis humani valititudinem, quum sit qualitas occulta talium effectuum productiua, sicut lapidem aquile a pregnante delatum per contactum virtualem ad caelum et ad matricem atque cotilidones aborsum prohibere a doctoribus asseritur ». 
à distance. Duns Scot explique ainsi que « le soleil selon sa puissance [potentia] est partout, où il n'est pas selon sa substance ». En réalité, pour lui, le seul contact vraiment indispensable est le « contact par le pouvoir ». Le contact par la substance n'est là que parce que les qualités actives de l'agent et les qualités passives du patient sont « inhérentes à leur substance matérielle quantifiée ». Duns Scot passe pour l'un des rares auteurs médiévaux à avoir accepté d'une certaine manière l'action à distance ${ }^{25}$.

Face à l'assertion selon laquelle « la vertu n'est pas là où la substance n'est pas » (Item, virtus non est ubi non est substantia), il répond précisément grâce à la distinction entre tactus virtualis et tactus mathematicus : « Le toucher mathématique n'est pas nécessaire à l'agent et au patient [...]. Donc est requis un toucher virtuel, et ainsi est compris Aristote, au VIIe livre de la Physique ${ }^{26}$.

Thomas d'Aquin avait distingué dans son Commentaire des Sentences (IV, d. 12, q. 1, art. 2, q. 1 arg. 2 et ad 2), le tactus mathematicus (applicable aux dimensions séparées, comme au lieu) et le tactus physicus (contact naturel entre corps sensible $)^{27}$. Duns Scot, lui, emploie l'expression tactus mathematicus pour définir le contact secundum superficies ${ }^{28}$ prôné au septième livre de la Physique, auquel il se réfère, c'est-à-dire à l'endroit où Aristote définit la nécessité d'un contact entre le moteur et ce qu'il meut. Duns Scot fait formellement allégeance au cadre contiguïste aristotélicien. Pourtant son tactus virtualis et le rôle déterminant qu'il lui confère dessine un tout autre cadre conceptuel, celui où l'action à distance est, pour ainsi dire, la règle.

Cela n'échappe pas à un autre tenant de l'action à distance mais dans une perspective radicalement différente : Guillaume d'Ockham.

\section{Guillaume d'Ockham}

Dans son commentaire au livre VII de la Physique, Ockham critique, sans le nommer, Duns Scot.

25 F. J. Kovach, "Action at a Distance in Duns Scotus and Modern Science », dans C. Bérubé (éd.), Regnum Hominis et Regnum Dei. Actus Quarti Congressus Scotistici Internationalis, Rome 1978, 477-490 (ici : 478-479).

26 Iohannes Duns Scotus, Quaestiones super libros Metaphysicorum Aristotelis, éds. R. Andrews, G. Etzkorn, G. Gál, R. Green, F. Kelley, G. Marcil, T. Noone et R. Wood, St. Bonaventure (NY) 1997, VIII, q. 3, $446:$ : [...] tactus mathematicus non est necessarius agentis et patientis [...]. Igitur requiritur tactus virtualis, et sic intelligitur Aristoteles, VII Physicorum ».

27 Cf. Deferrari, Barry, McGuiness, A Lexicon of St. Thomas Aquinas.

28 Kovach, «Action at a Distance », 479 note 6. 
[...] ils disent qu'il n'est pas toujours nécessaire que le moteur touche le mû par un toucher mathématique, mais par un toucher virtuel. Ainsi, ils disent que le Philosophe n'a pas voulu dire que le moteur et le mû étaient toujours ensemble localement, mais virtuellement ${ }^{29}$.

\section{Ockham réfute cette interprétation laxiste d’Aristote :}

Mais cette explication ne semble pas satisfaire à l'intention d'Aristote, parce qu'Aristote a expressément voulu dire qu'entre le moteur proche et ce qu'il meut il n'y a aucun intermédiaire corporel $^{30}$.

Et il ajoute plus loin qu'Averroès dans son commentaire sur la Physique, afin précisément de sauver cet axiome contiguïste, a expliqué l'attraction magnétique comme une altération du fer touché par la vertu magnétique, ce qu'il n'aurait pas été obligé de postuler si un contact virtuel était suffisant entre le fer et l'aimant $^{31}$. Ockham établit nettement l'incompatibilité de la thèse contiguïste avec celle faisant du contactus virtualis le contact nécessaire et suffisant pour remplir l'exigence de l'axiome aristotélicien.

En revanche, il ne revient pas pour autant à l'explication proposée par Averroès et suivie par tous les commentateurs latins de l'action de l'aimant sur le fer comme une altération du fer par la vertu magnétique. Fidèle à une causalité économique, il considère que la seule cause est celle sans laquelle (toutes choses égales) l'action n'aurait pas lieu ; la seule cause est donc l'aimant et il n'y a pas lieu de postuler une autre cause comme la vertu magnétique ${ }^{32}$. Ce faisant, il aboutit à une forme d'action à distance beaucoup plus radicale que celle, modérée, de Duns Scot.

29 Guillelmus de Ockham, Expositio in libros Physicorum Aristotlelis, VII.3, éds. R. Wood, R. Green, G. Gál, J. Giermek, F. Kelley, G. Leibold et G. Etzkorn, St. Bonaventure (NY) 1985, 621, note $\S 2.1$ (renvoi à Duns Scot) : « Pro istis et consimilibus difficultatibus dicunt quod non semper oportet movens tangere motum tactu mathematico sed tactu virtuali. Unde dicunt quod Philosophus non intendit quod movens sit simul cum moto localiter, sed virtualiter ».

30 Ibid., 621 : «Sed ista expositio non videtur sufficere pro intentione Aristotelis, quia Aristoteles expresse intendit quod inter movens propinquum et motum nullum est medium corporale ». 31 Ibid., 622 : « Item Commentator, eodem commento, ad salvandum illam propositionem, dicit quod ferrum alteratur a magnete ad aliquam qualitatem quae movet ferrum, quod tamen non oporteret ponere si sufficeret talis contactus virtualis ».

32 A. Goddu, «William of Ocham's Arguments for Action at a Distance », Franciscan Studies 44 (1984), 227-244. 


\section{Petrus Turisanus}

À peu près à la même époque, au tournant des XIII ${ }^{\mathrm{e}}$ et XIV ${ }^{\mathrm{e}}$ siècles, le médecin Pietro Torrigiano (Turisanus) (v. 1275-v. 1330) dans son commentaire à l'Art médical de Galien, le Plusquam commentum in Parvam Galeni artem, lorsqu'il commente la distinction d'Avicenne entre trois types d'attraction (par la forme spécifique, par le vide et par la chaleur) ${ }^{33}$, écrit à propos des opérations procédant de l'attraction par la forme substantielle (c'est-à-dire les propriétés occultes), comme le fer attiré par l'aimant, ou le poisson torpille paralysant la main du pêcheur tenant le filet :

[...] de telles actions ne réclament pas nécessairement un contact de l'agent avec le patient, comme l'est le contact de quantité (magnitudinis), mais un contact de vertu. Ce ne sont pas en effet des actions naturelles, mais virtuelles, qui sont dites surnaturelles [... $]^{34}$.

Dans ce passage, Turisanus, contredit la tradition dominante de la philosophie naturelle et de la médecine sur deux points. D'une part, il renvoie l'attraction par la forme substantielle (c'est-à-dire par les propriétés occultes) vers le surnaturel, alors que l'interprétation généralement donnée est strictement naturelle ${ }^{35}$. D’autre part, il défend une forme d'action à distance. Il précise son point de vue :

Et le contact naturel n'exige pas le contact de l'agent avec le patient, lequel est un contact de quantité (magnitudinis), mais [il exige un contact] métaphorique, qui est dit contact de vertu, raison pour laquelle de telles actions sont dite virtuelles et d'une certaine manière surnaturelles, mais il réclame seulement la présence d'un patient qui, par la propriété de sa forme, est susceptible de recevoir un tel acte ${ }^{36}$.

33 D. Jacquart, La Médecine médiévale dans le cadre parisien, $X I V^{e}-X V^{e}$ siècle, Paris 1998, 368. 34 Turisanus, Plus quam commentum in Parvam Galeni artem, Venise 1557, III, f. 151v : « Propter quod tales actiones non expetunt necessario contactum agentis ad patiens, qualis est contactus magnitudinis, sed virtutis. Non enim sunt actiones naturales, sed virtuales, quae supernaturales dicuntur ». (Une autre édition : Trusiani [sic !] monaci cartisiensis Plusquam commentum in librum Galeni qui microtechni intitulatur, Bologne 1489, sign. A ii va, donne une leçon erronée : " qualis est contactus magnitudinis et virtutis »).

35 N. Weill-Parot, Points aveugles de la nature : la rationalité scientifique médiévale face à l'occulte, l'attraction magnétique et l'horreur du vide (XIII - milieu du XV siècle), Paris 2013.

36 Turisanus, Plus quam commentum in Parvam Galeni artem, f. 152r : « Neque etiam expetitur naturalis contactus activi ad passivum, qualis est contactus magnitudinis, sed metaphoricus, qui dicitur contactus virtutis, propter quod tales actiones virtuales dicuntur et quodammodo supernaturales, sed expetitur [experitur] tantum praesentia passivi, quod ex proprietate sue formae talis actus susceptivum existit ». 
Cette qualification du contact virtuel de contact métaphorique se trouve déjà chez Thomas d’Aquin. Dans son Commentaire des Sentences (I, d. 37, q.3, art. 1, co.), l'Aquinate distingue deux contacts : le toucher à proprement parler, dans lequel les extrémités sont ensemble, et le toucher métaphorique, qui est à travers une action et qui seul convient à l'ange ${ }^{37}$.

\section{Les species}

Dès le milieu du XIV ${ }^{\mathrm{e}}$ siècle, partir de Jean Buridan et Albert de Saxe, l'action apparemment à distance trouve sa solution définitive dans le modèle contiguïste pour des cas comme l'attraction magnétique ou la torpille paralysant la main du pêcheur à travers le filet (ou le trident), grâce à la doctrine des species provenant de la perspectiva. La vertu de l'aimant émet autour d'elle des species qui se multiplient de proche en proche dans l'air intermédiaire et entrent ainsi au contact du morceau de fer ${ }^{38}$.

En inscrivant son propre commentaire à la Physique dans ce modèle explicatif saxo-buridanien qui fut très influent notamment dans les nouvelles universités d'Europe centrale ${ }^{39}$, Benedictus (Benedykt) Hesse de Cracovie (v. 1349-1456) explique que, dans l'attraction magnétique, le moteur et le mobile sont bien ensemble "virtuellement », mais, pour lui, cette conjonction virtuelle n'est en rien une action à distance ${ }^{40}$. Il rejette la thèse de son opponens qui prétendrait

37 S. Thomae Aquinatis Scriptum super libros Sententiarum, t. 1, éd. P. Mandonnet, Paris 1929, I, d. 37 q. 3 art. 1, Resp., 870 : «Contactus autem dicitur dupliciter : proprie et metaphorice. Proprie tangere est, habere ultima simul ; et patet quod hoc angelo convenire non potest. Tactus autem metaphoricus est per actionem, sicut dicitur contristans tangere ; et iste tactus angelo potest convenire. Relinquitur ergo quod angelus definiri vel determinari non potest ad locum aliquem, nisi per actionem et operationem ».

38 Weill-Parot, Points aveugles de la nature, 209-227. Sur la doctrine des species, voir supra note 16. 39 Voir notamment : J. Hamesse, «L'importance de l'étude d'Aristote dans les universités médiévales allemandes. Le témoignage des manuscrits conservés à la bibliothèque d'Erfurt », dans J. A. Aertsen (éd.), Die Bibliotheca Amploniana, Berlin-New York 1995, 54-72.

40 Benedictus Hesse, Quaestiones super octo libros "Physicorum" Aristotelis, éd. S. Wielgus, Wrocław 1984, VII, 653 : « De alio : Inter magnetem et ferrum est quaedam convenientia et proportio complexionis, per quam ferrum est susceptivum influentiae magnetis seu virtutis influxae a magnete, et sic adamas attrahit ad se ferrum, et ibi tunc movens adhuc est simul cum moto virtualiter, quia per influxum multiplicatur a magnete ». Un autre maître, Laurent de Lindores (v. 1372-v. 1437), a effectué un commentaire qui présente de nombreux points communs avec celui de Benedykt Hesse, y compris dans ce passage (les parallèles entre les deux commentaires sont aussi relevés dans l'édition de S. Wielgus). Sur Laurent de Lindores : T. Dewender, Das 
qu'il défend un modèle explicatif où « un accident migrerait de sujet en sujet », alors que, selon lui, il faut imaginer que « l'influence multipliée par l'aimant génère » une influence qui en génère une autre de proche en proche jusqu'au morceau de fer, comme la pierre jetée dans l'eau qui entraîne une production successive de cercles dans l'eau ${ }^{41}$.

De ce point de vue, cette conjonction virtuelle des deux corps se trouve lavée du soupçon d'action à distance. Dans d'autres passages, c'est encore au contact virtuel que Benedictus Hesse se réfère pour qualifier le contact entre l'intelligence motrice et le corps céleste ou bien l'action du ciel sur la volonté humaine ${ }^{42}$.

\section{L’âme, l'aimant et la vertu}

En somme, plusieurs auteurs médiévaux ont mis en avant la notion de contact virtuel entre une substance spirituelle (âme, ange, Dieu ou démon) et un corps, en pensant ainsi maintenir ce type d'action dans le cadre contiguïste voulu par Aristote entre le moteur et ce qu'il meut. Pourtant, on retrouve ce concept appliqué, dans le monde physique, à des cas d'action apparemment à distance, entre le corps céleste et le corps terrestre distant, ou bien même entre deux corps sublunaires. Les témoignages désaccordés de Duns Scot, Guillaume d'Ockham et Turisanus montrent qu'au tournant des XIII ${ }^{\mathrm{e}}$ et XIV ${ }^{\mathrm{e}}$ siècles, ce contact virtuel appliqué aux

Problem des Unendlichen im ausgehenden 14. Jahrundert : Eine Studie mit Textedition zum Physikkommentar des Lorenz von Lindores, Amsterdam-Philadelphie 2002, 7-10 ; Id., « Lawrence of Lindores and His Commentaries on Aristotle », dans J. Marenbon (éd.), Aristotle in Britain during the Middle Ages, Turnhout 1996, 323-335).

41 Benedictus Hesse, Quaestiones super octo libros "Physicorum" Aristotelis, 653 : "Sed dubium : Si adamas attraheret ferrum isto modo, sequitur, quod accidens migraret de subiecto in subiectum, quia ista influentia intrat aërem et postea in ferrum. Respondetur : Non est imaginandum quod ista influentia multiplicaretur ab adamante in aërem et ab aëre in ferrum, sed imaginandum est, quod influentia mutliplicata ab adamante generat unam aliam et alia aliam usque ad ferrum, ut patet de lapide proiecto in aquam, ubi unus circulus generat alium ».

42 « Dicitur, quod intelligentia appropriata suo orbi, licet non est simul cum orbe per contactum quantitativum, tamen bene per contactum virtualem. Sed si dicitur : "Tamen incorporeum non est simul cum corporeo, nec distat ab eo”, respondetur : Hoc est verum quantitative, non autem secundum contactum virtualem. (Ibid., VII, q. 9, 654-655) ; Utrum caelum agat in intellectum et voluntatem humanam « [...] De alio quo dicitur : “Omnis actio fit per contactum”, respondetur : verum est de actione, ubi agens immediate agit in passum. Modo sic non est de caelo et anima intellectiva. Vel dicitur, quod caelum adhuc agit in intellectum per contactum, licet non formalem tamen virtualem » (Ibid., VIII, q. 10, 713). 
corps a pu être conçu comme une action proprement à distance, même si ce ne fut pas toujours le cas.

Est-ce à dire qu'implicitement l'action de l'esprit sur le corps est conçu comme une action à distance qui n'aurait que métaphoriquement l'apparence d'un contact ? Il n'y a pas lieu ici d'examiner la question du pouvoir distant de l'âme, que récemment Vittoria Perrone Compagni, Béatrice Delaurenti et Aurélien Robert notamment ont explorée ${ }^{43}$; il suffira ici d'évoquer la réémergence au XVI siècle de l'hypothèse (exclue au Moyen Âge) d'une animation de l'aimant.

Il s'agit d'une thèse attribuée par Aristote à Thalès dans le De anima. Les Jésuites de Coimbra (1543-1592) dans leur commentaire à la Physique évoquent cette thèse selon laquelle c'est parce que « l'aimant serait animé qu'il attirerait le fer, à savoir en lui communiquant la vie ». La thèse, expliquent les Jésuites de Coimbra, ne tient pas $^{44}$ :

L'aimant, en effet, n'est pas puissant par une âme, puisqu'il ne croît pas ou ne donne pas d'autre indice d'une fonction vitale ; et s'il était doté d'une âme, cela ne serait pas suffisant pour faire participer le fer à la vie et s'emparer de ce dernier ${ }^{45}$.

En somme, à moins d'attribuer une capacité propre à l'âme à se déployer à distance l'animation de l'aimant ne résout rien. Après Cardan, c'est William Gilbert qui promut cette doctrine de l'animation de l'aimant dans son De magnete (1600). Mais il s’inscrivait dans une perspective philosophique dès lors largement en

43 Voir, par ex., V. Perrone Compagni, « “Artificiose operari”. L’immaginazione di Avicenna nel dibattito medievale sulla magia », dans M. Bettetini et F. Paparella (éds.), collab. R. Furlan, Immaginario e immaginazione nel medioevo, Louvain-la-Neuve 2009, 271-296 ; B. Delaurenti, La Puissance des mots. "Virtus verborum ». Débats doctrinaux sur le pouvoir des incantations au Moyen Âge, Paris 2007 ; Ead., La Contagion des émotion. Compassio, une énigme médiévale, Paris 2016 ; A. Robert, « Dino del Garbo et le pouvoir de l'imagination sur le corps », Archives d'histoire doctrinale et littéraire du Moyen Âge 81 (2014), 139-195 ; Id., " Le pouvoir des incantations selon les médecins du Moyen Âe (XIII ${ }^{\mathrm{e}}-\mathrm{XV}^{\mathrm{e}}$ siècle) », dans N. Bériou, J.-P. Boudet et I. Rosier-Catach (éds.), Le Pouvoir des mots au Moyen Âge, Turnhout 2014, 459-489. Qu'il me soit aussi permis de renvoyer à : N. Weill-Parot, « Pouvoirs lointains de l'âme et des corps : l'action à distance entre philosophie et magie, entre Moyen Âge et Renaissance », Lo Sguardo. Rivista Elettronica di Filosofia 10 (2012), 85-98 (http://www.losguardo.net).

44 Collegium Conimbricensis societatis Jesu, Commentarii in octo libros Physicorum, Lyon 1625, VII, cap. 2, q. 1, art. 3, 168-169: « Thales referente Aristotele I. de anima cap. 2 text. 32 pronunciavit magnetem propterea quod animatus esset, ferrum allicere, impertita ei videlicet vita : quem videtur secutus fuisse Porphyrius in libro de abstinentia ab animantibus. Sed hi perspicue errant».

45 Ibid. : «Nam neque magnes animo pollet, cum neque augescat, aut aliud vitalis functionis specimen det. Nec si anima polleret, id ad impertiendam vitam, et corripiendum ferrum sat esset ». 
rupture avec l'aristotélisme, et dans un modèle néoplatonisant l'âme pouvait acquérir aisément la capacité d'agir à distance ${ }^{46}$.

Le détour par les actions d'un corps sur un autre, induit par l'usage de la notion de contactus virtualis, permet donc de comprendre que l'opération d'une substance spirituelle sur un corps tendait vers une action à distance - type d'action pourtant rejeté dans le cadre contiguïste aristotélicien. C'est sans doute l'un des aspects de cette difficulté à inscrire l'interaction entre l'esprit et le corps dans un modèle explicatif qui soit général et, pour ainsi dire, homothétique par rapport au modèle contiguïste appliqué aux corps.

46 N. Weill-Parot, " Magnetic Attraction as a Challenge to the Inanimate Realm. The Example of Walter Burley » dans D. Jacquart et N. Weill-Parot (éds.), Substances minérales et corps animés / Mineral Substances and Animate Bodies (1100-1150). De la philosophie de la matière aux pratiques médicales, Paris 2012 (" Histoire des savoirs »), 87-110 ; Id., "О значении одной отвергнутой гипотезы в Средневековье и эпоху Ренессанса: одушевленность магнита », іп Павел Соколов (ред.), Многоликая софистика: нелегитимная аргументация в интеллектуальной культуре Европы Средних веков и раннего Нового времени, Москва, 2015, 316-329 [= « La fonction d'une hypothèse refoulée entre Moyen Âge et Renaissance : l'animation de l'aimant » [traduit par Maria Sorokina], dans P. Sokolov (éd.), La Sophistique aux mutiples visages : l'argumentation illégitime dans la culture intellectuelle de l'Europe au Moyen Âge et au début de l'époque moderne, Moscou 2015, 316-329]. 